Ergod. Th. \& Dynam. Sys. (1986), 6, 259-264

Printed in Great Britain

\title{
On Denjoy's theorem for endomorphisms
}

\author{
I. MALTA
}

Departamento de Matemática, Pontifícia Universidade Católica do Rio de Janeiro, Rua Marquês de São Vicente, 225, 22453-Rio de Janeiro-Brasil

(Received 6 March 1984 and revised 11 December 1984)

Abstract. In this paper we give an extension to $C^{2}$ endomorphisms of the circle of the well known Denjoy's theorem on $C^{2}$ diffeomorphisms. We also give a simple proof of an extension of Block and Franke's theorem on the existence of periodic points for maps of the circle.

\section{Introduction}

One of the fundamental results in one-dimensional dynamics is Denjoy's theorem: THEOREM (Denjoy [3]). If $f$ is a $C^{2}$ diffeomorphism of the circle $S^{1}=\mathbb{R} / \mathbb{Z}$ then either $f$ is topologically conjugate to an irrational rotation or it has periodic points.

The aim of this paper is to prove a similar result for endomorphisms of $S^{1}$. Let $\operatorname{End}^{r}\left(S^{1}\right)$ be the set of endomorphisms of class $C^{r}, r \geq 0$, of $S^{1}$ and let $\operatorname{End}_{k}^{r}\left(S^{1}\right)$ be the set of $C^{r}$-endomorphisms of degree $k$. Recall that a point $z \in S^{1}$ is a critical point of an endomorphism $f \in \operatorname{End}^{1}\left(S^{1}\right)$ if $f^{\prime}(z)=0$. We shall say that $z$ is a non-flat critical point if there exists an integer $k>1$ such that $f$ is $C^{k}$ in a neighbourhood of $z$ and $f^{(k)}(z) \neq 0$.

The first extension of Denjoy's theorem is due to Yoccoz [7]:

THEOREM (Yoccoz). If $f$ is a $C^{2}$ homeomorphism of $S^{1}$ all of whose critical points are non-flat, then either $f$ is topologically conjugate to an irrational rotation or it has periodic points.

The non-flatness hypothesis is impossible to avoid because of the example constructed by G. R. Hall [4] of a $C^{\infty}$ homeomorphism of $S^{1}$ that has no periodic points and is not topologically equivalent to a rotation. Anyway, it is a reasonably weak assumption, satisfied generically. It is also satisfied by any real analytic endomorphism of the circle.

In order to state our main result, let us denote by $P(f), R(f)$ and $\Omega(f)$ respectively, the sets of periodic, recurrent and non-wandering points of $f$.

THEOREM A. If $f$ is a $C^{2}$ endomorphism of the circle and all its critical points (if any) are non-flat then either $f$ is topologically conjugate to an irrational rotation or $\overline{P(f)}=$ $\overline{R(f)}$. 
The results used to prove theorem A enable us to give a simple proof of an extension of Block and Franke's theorem [2] on the existence of periodic points for maps of the circle. If $z \in S^{1}$ and $f \in \operatorname{End}^{0}\left(S^{1}\right)$, let $I$ be the maximal interval containing $z$ such that $\left.f\right|_{I}$ is constant. We say that $z$ is a turning point if $f(z)$ is an extreme value of $f$ in a neighbourhood of $I$.

THEOREM B. Let $f \in \operatorname{End}_{1}^{2}\left(S^{1}\right)$ and suppose that all non-turning critical points of $f$ are non-flat. If $f$ has a turning critical point, then $f$ has periodic points.

We also provide a simple example which shows that theorem $\mathrm{A}$ is not true if we replace the recurrent set $R(f)$ by the non-wandering set $\Omega(f)$ of $f$.

I would like to thank J. Gheiner, M. J. Pacífico and J. C. Yoccoz for useful talks on this subject.

\section{Statements and proofs}

Let $f$ be an endomorphism of $S^{1}$. A lift for $f$ is a function $F: \mathbb{R} \rightarrow \mathbb{R}$ such that $f \Pi=\Pi F$, where $\Pi: \mathbb{R} \rightarrow S^{1}=\mathbb{R} / \mathbb{Z}$ is the canonical projection. If $\operatorname{deg}(f)$, the degree of $f$, is $k$ then $F(x+1)=F(x)+k$ for every $x$.

To prove theorem A when $P(f)=\varnothing$, we use Yoccoz's theorem and the following result due to Auslander and Katznelson [1], which is an extension to continuous endomorphisms of a well known theorem of Poincaré [6].

PROPOSITION 1. If $f$ is a continuous endomorphism of the circle with no periodic points, then $f$ is semi-conjugate to an irrational rotation, i.e. there exists a continuous degree one map $h: S^{1} \subseteq$ and an irrational rotation $R$ of the circle such that $R h=h f$. Moreover, the semi-conjugacy $h$ is monotone.

Let $f \in$ End $^{0}\left(S^{1}\right)$ with $P(f)=\varnothing$ and $h: S^{1} \circlearrowleft$ be the semi-conjugacy given by proposition 1. We say that a non-degenerate interval $I \subset S^{1}$ is a plateau if $h$ is constant in $I$ and $I$ is a maximal interval with this property. That is, if $J \supset I$ and $h$ is constant in $J$ then $J=I$. It follows immediately from proposition 1 that:

(i) The plateaux depend only on $f$.

(ii) If $I=[a, b]$ is a plateau, then $f(I)=[f(a), f(b)]$ is also a plateau.

(iii) If $A$ is the union of the interior of the plateaux, one has $\Omega(f)=R(f)=S^{1} \backslash A$, which is $S^{1}$ or a Cantor set.

With these results we prove the following lemma:

LeMMA 1. Let $f \in \operatorname{End}^{1}\left(S^{1}\right)$ be such that $P(f)=\varnothing$ and $R(f)$ does not contain flat critical points. Then there exists a homeomorphism $g \in \operatorname{End}_{1}^{1}\left(S^{1}\right)$ such that $R(g)=R(f)$ and all its critical points are non-flat.

Proof. The non-flatness hypothesis implies that the critical points in $R(f)$, if any, are isolated amongst critical points so there are at most finitely many plateaux 
having critical points of $f$. From (ii) we can modify $f$ in these plateaux in order to obtain $g$ with the required properties.

We now prove theorem $B$.

Proof of theorem B. Suppose $P(f)=\varnothing$. Then proposition 1 applies and since $f$ has a turning point we conclude that $R(f)$ is a proper subset of $S^{1}$. On the other hand, from (ii), (iii) and the monotonicity of the semi-conjugacy given by proposition 1 , we conclude that any critical point in $R(f)$ cannot be a turning point. So $R(f)$ does not contain flat critical points. Now, lemma 1 gives a contradiction with Yoccoz's theorem.

To prove theorem A, we need one more result, which is a version for continuous endomorphisms of the circle of Young's theorem [8] that states that $\overline{P(f)}=\overline{R(f)}$ holds for any piecewise monotone map of the interval. The version we present here evolved from discussions during the expositions of J. Gheiner on Young's theorem in a seminar on maps of the circle and the interval at IMPA.

Let $f \in \operatorname{End}^{0}\left(S^{1}\right)$. A set $\left\{c_{0}, \ldots, c_{k}=c_{0}\right\}$ will be called a partition by turning points of $f$ if $c_{j}$ is a turning point of $f$ and the restriction of $f$ to the interval $I_{j}=\left[c_{j-1}, c_{j}\right]$ is a monotone map, for $1 \leq j \leq k$.

The following lemma can be proved directly from lemmas 1-5 in $\S 2$ of [8].

LEMMA 2. If $f \in \operatorname{End}^{0}\left(S^{1}\right)$ and there exists a partition $\left\{c_{0}, \ldots, c_{k}\right\}$ by turning points of $f$ such that $c_{j} \in \Omega(f)$ for $0 \leq j \leq k$, then $\overline{P(f)}=\overline{R(f)}$.

Proposition 2. If $f \in \operatorname{End}^{0}\left(S^{1}\right)$ is a piecewise monotone endomorphism, then either $P(f)=\varnothing$ or $\overline{P(f)}=\overline{R(f)}$.

Proof. We will modify $f$ in such a way to obtain $g \in \operatorname{End}^{0}\left(S^{1}\right)$ with the following properties:

(i) $g=f$ on $R(f)$;

(ii) $g \neq f$ at most only on a finite union of open intervals $\bigcup_{i=1}^{k} J_{i}$ and $\left.g\right|_{J_{i}}$ is constant for $1 \leq i \leq k$;

(iii) $P(g) \backslash P(f)$ has at most finitely many points.

In fact if $z_{0} \in P(g) \backslash P(f)$ then the orbit of $z_{0}, O\left(z_{0}, g\right)$ needs to intersect $J_{i}$ for some $i$. We may assume that $z_{0} \in J_{i}$ and $g^{n}\left(z_{0}\right)=z_{0}$. Then if $z \in J_{i}$ we have that $g^{n}(z)=z_{0}$, that is, $z$ is eventually periodic but not periodic. Thus to each $z \in$ $P(g) \backslash P(f)$ we can attach one $J_{i}$ and there are only finitely many $J_{i}$ 's.

(iv) Either there is a partition by turning points $\left\{c_{0}, \ldots, c_{k}\right\}$ with $c_{i} \in \Omega(g)$ for $0 \leq i \leq k$ or $g$ is monotone.

Let us construct $g$. If $f$ is monotone, take $g=f$. Otherwise, let $z_{0} \in R(f)$ and $a_{0} \in[0,1]$ be such that $\Pi\left(a_{0}\right)=z_{0}$. Let $F$ be a lift of $f$ and let $a_{0} \leq x_{1}$ be, amongst turning points, the smallest turning point of $F$. If $x_{1}$ is not isolated, let $I_{1}=\left[x_{1}, y_{1}\right]$ the maximal interval such that $\left.F\right|_{I_{1}}$ is constant.

If $\Pi\left(I_{1}\right) \cap R(f) \neq \varnothing$, do nothing. Note that this is the case if $x_{1}=a_{0}$. If not, suppose that it is a maximum. 


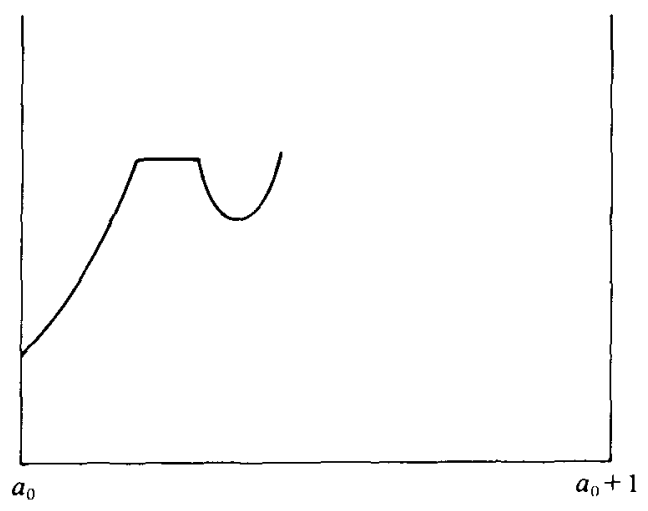

FIGURE 1

\section{Push down $F$ on $I_{1}$ until}

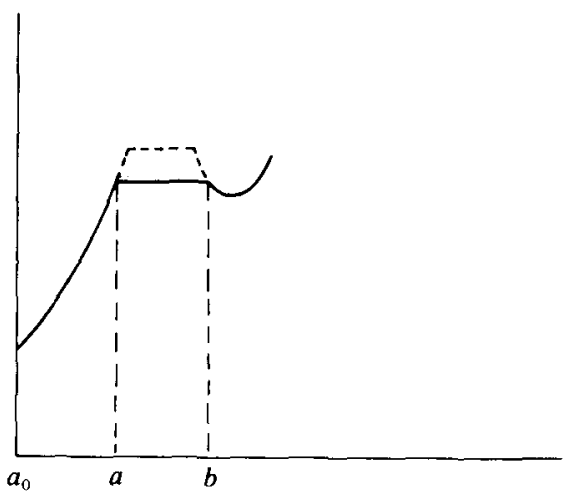

Figure 2

$\Pi(a)$ or $\Pi(b)$ belongs to $\overline{R(f)}$, or

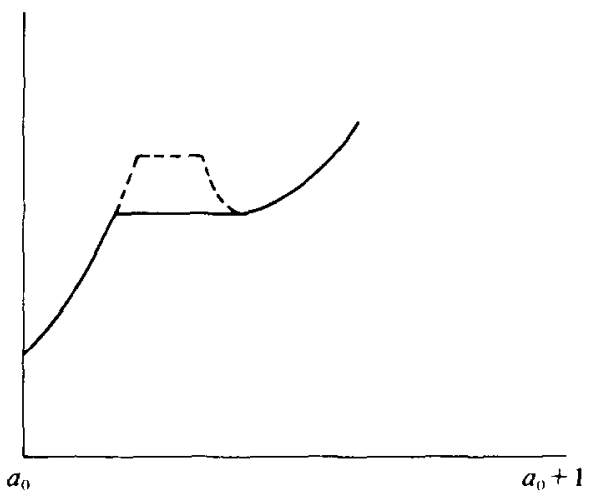

FIGURE 3 
the second turning point (or turning interval) disappears. Now work in the same way on the next turning point: $x_{2}$, if figure 2 prevails and $x_{3}$ if figure 3 prevails. Since $F$ is piecewise monotone, after a finite number of such steps we obtain a function $G$ such that $\operatorname{deg}(G)=\operatorname{deg}(F)$. (Note that we never touch $a_{0}$ and $a_{0}+1$, so

$$
G\left(a_{0}+1\right)=F\left(a_{0}+1\right)=F\left(a_{0}\right)+\operatorname{deg}(F)=G\left(a_{0}\right)+\operatorname{deg}(F) \text {. }
$$

Let $g$ be the projection of $G$ to $S^{1}$. Clearly $g$ satisfies properties (i)-(iv) and so it suffices to prove that $\overline{P(g)}=\overline{R(g)}$ or $P(g)=\varnothing$.

If $g$ is not monotone, (iv) and lemma 2 give the result. Suppose then that $g$ is monotone. If $\operatorname{deg} g=1$ there are two possibilities: the rotation number of $g$ is a rational or an irrational number. In the first case $P(g)=R(g)$; in the second $P(g)=\varnothing$ and so $P(f)=\varnothing$. If $\operatorname{deg} g=-1$ then $\operatorname{deg} g^{2}=1$ and $g^{2}$ has a fixed point, and so $P(g)=R(g)$. There remains the case where $|\operatorname{deg} g| \geq 2$. In this case we know, from [5], that if $\phi_{k}: S^{1} \varsigma$ is defined by $\phi_{k}(z)=z^{k}$, where $k=\operatorname{deg} g$, then $g$ is semi-conjugate to $\phi_{k}$ by a monotone continuous map $h: S^{1} \varsigma$ of degree one. To prove that $\overline{P(g)}=$ $\overline{R(g)}$ let $z_{0} \in R(g)$ and suppose that $z_{0} \notin P(\mathrm{~g})$. Then, given any neighbourhood $V$ of $z_{0}$, there exists an interval $J \subset V$ with non-empty interior, such that $z_{0}$ is an end point of $J$ and if $z_{1}$ is the other end point, then $h\left(z_{0}\right) \neq h(z) \neq h\left(z_{1}\right)$ for every $z \in$ int $J$. This implies that $h(J)$ is an interval with non-empty interior and if $w \in h(J)$, then $h^{-1}(w) \subset J$. Now, let $w_{0} \in h(J)$ such that $w_{0}$ is a periodic point of $\phi_{k}$. Then $h^{-1}\left(w_{0}\right) \cap$ $P(g) \neq \varnothing$ and so $P(g) \cap V \neq \varnothing$.

Proof of theorem A. The proof goes by contradiction. From proposition 2 it suffices to prove that if $P(f)=\varnothing$ then $f$ is conjugate to an irrational rotation. If $f$ has no periodic points proposition 1 says that $f$ is semi-conjugate to an irrational rotation $R$. Suppose, by contradiction, that $f$ is not conjugate to $R$. Then $S^{\mathbf{l}} \backslash R(f) \neq \varnothing$ and from lemma 1 we conclude that there exists a homeomorphism $g$ of class $C^{2}$ having no flat critical points such that $R(g)=R(f)$ which contradicts Yoccoz's theorem.

The following example, which is a simple adaptation of the one given by L. S. Young in [8], shows that theorem $\mathrm{A}$ is not true if we replace the recurrent set $R(f)$ by the non-wandering set $\Omega(f)$.

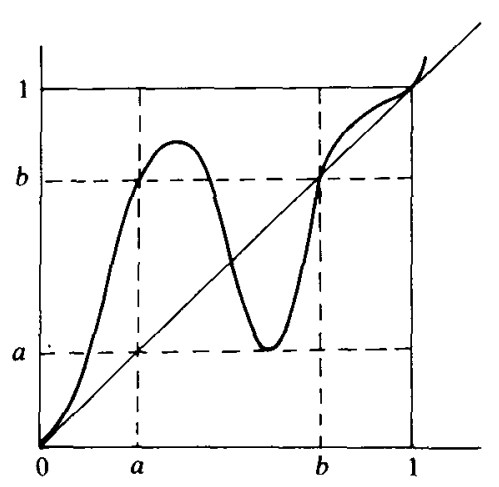

Figure 4 


\section{REFERENCES}

[1] J. Auslander \& Y. Katznelson. Continuous maps of the circle without periodic points. Israel J. Math. 32, No. 4 (1974), 375-381.

[2] L. Block \& J. Franke. Existence of periodic points for maps of $S^{\mathbf{1}}$. Invent. Math. 22 (1973), 69-73.

[3] A. Denjoy. Sur les courbes définies par les équations différentielles à la surface du tore. J. Math. Pures Appl. 11 (1932), 333-375.

[4] G. R. Hall. A $C^{\infty}$ Denjoy counter-example. Ergod. Th. \& Dynam. Sys. 1 (1981), 261-272.

[5] Z. Nitecki. Differentiable Dynamics: An Introduction to the Orbit Structure of Diffeomorphisms. M.I.T. Press, 1971.

[6] H. Poincaré. Oeuvres Complètes. t.l.Gauthier-Villars, Paris, 1952, pp. 137-158.

[7] J. C. Yoccoz. Il n'y a pas de contre-exemple de Denjoy analytique. In C.R.A.S. 298 (1984).

[8] L. S. Young. A closing lemma on the interval. Invent. Math. 54 (1970), 179-187. 